\title{
Biskyrmion lattices in centrosymmetric magnetic films
}

\author{
Daniel Capic (), Dmitry A. Garanin, and Eugene M. Chudnovsky (1) \\ Physics Department, Herbert H. Lehman College and Graduate School, The City University of New York, \\ 250 Bedford Park Boulevard West, Bronx, New York 10468-1589, USA
}

(Received 19 July 2019; published 8 October 2019)

\begin{abstract}
A theoretical framework is developed that permits construction of biskyrmion lattices observed in nonchiral magnetic films. Skyrmion and antiskyrmion configurations are discussed. We study films of finite thickness containing up to $1000 \times 1000 \times 100$ spins. Hexatic biskyrmion lattices in a pure two-dimensional (2D) exchange model are naturally described by the Weierstrass $\wp$ and $\zeta$ elliptic functions. Starting with such a lattice as an initial state, we investigate how it evolves toward a minimum-energy state in a zero magnetic field in the presence of perpendicular magnetic anisotropy (PMA) and dipole-dipole interaction. Metastable biskyrmion lattices exist at low PMA. At higher PMA, we observe stable triangular lattices of biskyrmion bubbles containing Bloch lines, whose energies are lower than the energy of the uniformly magnetized state.
\end{abstract}

DOI: 10.1103/PhysRevResearch.1.033011

\section{INTRODUCTION}

Skyrmions were initially introduced in nuclear physics as solutions of the nonlinear $\sigma$ model that can describe atomic nuclei [1-3]. They possess topological charge: $Q= \pm 1, \pm 2$, etc. $Q=1$ would correspond to a single nucleon, $Q=2$ could describe a deuteron [4,5], $Q=4$ would provide a model of an $\alpha$ particle, etc.

In magnetic films, skyrmions are defects of the ferromagnetic order. Their topological charge $Q$ arises from different homotopy classes of the mapping of a three-component fixed-length magnetization field onto the $x y$ plane. Skyrmions represent a very active field of research due to their nanoscale size and potential for a dense topologically protected data storage and information processing [6-11]. Much bigger micron-size magnetic bubbles intensively studied in $1970 \mathrm{~s}$ $[12,13]$ possessed similar topological properties. They were cylindrical domains surrounded by narrow domain walls. On the contrary, a typical skyrmion would be small compared to the domain wall thickness, making it conceptually similar to the topological objects studied in nuclear physics $[14,15]$.

Research on magnetic skyrmions focuses on their stability and dynamics. Magnetic bubble lattices are in effect domain structures $[12,13,16,17]$ stabilized by the perpendicular magnetic anisotropy (PMA), dipole-dipole interaction (DDI), and the external magnetic field. On the contrary, in the absence of other interactions, small skyrmions collapse [18] due to violation of the scale invariance of the two-dimensional (2D) exchange model by the atomic lattice. They can be stabilized by the Dzyaloshinskii-Moriya interaction that is present in materials lacking inversion symmetry (DMI) $[9,19-21]$ or by

Published by the American Physical Society under the terms of the Creative Commons Attribution 4.0 International license. Further distribution of this work must maintain attribution to the author(s) and the published article's title, journal citation, and DOI. quenched randomness [22,23] that has an effect similar to that of the DMI.

While $Q=1$ skyrmions and lattices of such skyrmions have been intensively studied, observation of $Q=2$ biskyrmions (see Fig. 1), and biskyrmion lattices has been rare. Unlike skyrmions, that have been mostly observed in chiral films, stable biskyrmions lattices have been initially reported down to zero field in two nonchiral films of sufficient thickness: the $\mathrm{La}_{2-2 x} \mathrm{Sr}_{1+2 x} \mathrm{Mn}_{2} \mathrm{O}_{7}$ manganite [24] and the $\left(\mathrm{Mn}_{1-x} \mathrm{Ni}_{x}\right)_{65} \mathrm{Ga}_{35}$ half-Heusler alloy [25]. Recent works [26-28] provided further experimental and numerical evidence of stable biskyrmions in centrosymmetric magnetic materials.

Theoretical works on biskyrmions have been scarce. It has been shown [29] that topological defects with $Q>1$ appear naturally in centrosymmetric films due to the presence of Bloch lines in labyrinth domains on increasing magnetic field. Numerical investigation of biskyrmions, including the current-induced dynamics, was performed in Ref. [30] within a 2D frustrated micromagnetic model. Biskyrmions arising from frustrated Heisenberg exchange have also been reported in the studies of triangular spin lattices [31] and in a model based upon Ginzburg-Landau theory [32]. Metastable biskyrmion configurations have been observed in LandauLifshitz dynamics of a frustrated bilayer film [33]. However, the study of biskyrmion lattices has been absent so far.

In real systems, the magnetic biskyrmions are more complicated than generic $Q=2$ solutions of the Belavin-Polyakov (BP) 2D exchange model. They are formed by a number of competing interactions on top of the exchange, such as PMA and DDI. The latter rules out any meaningful analytical solution. In this paper, we adopt the following approach. First, we prepare a biskyrmion lattice that is a solution of the pure exchange model. Fortunately, it can be described in the complex plane, $z=x+i y$, by standard elliptic Weierstrass functions that have been previously used to build multiskyrmion configurations in nuclear physics [3]. We then turn on the PMA and DDI and compute numerically evolution of the 


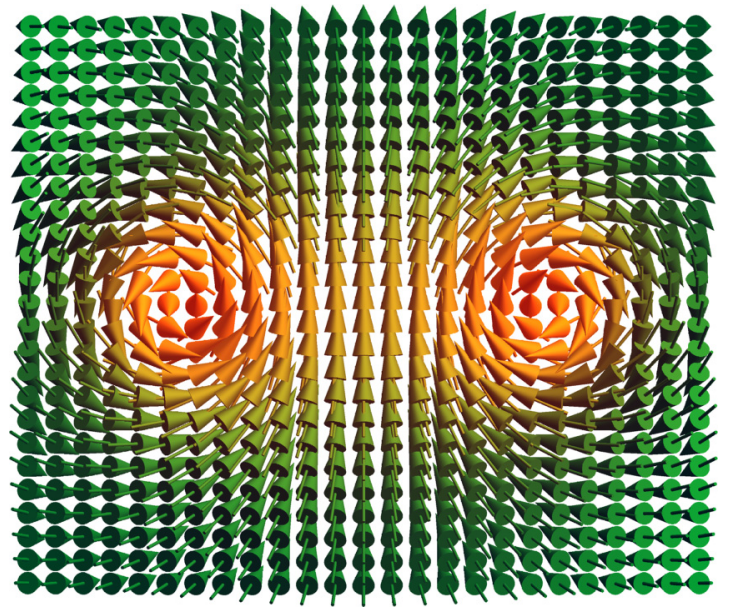

FIG. 1. Computer-generated spin field in a Bloch-type biskirmion in a ferromagnetic film.

system toward the spin configuration that corresponds to the energy minimum.

Our calculations are performed on 3D lattices containing $1000 \times 1000 \times 100$ spins that describe magnetic films of finite thickness. In accordance with real experiments, we find that the lowest energy achieved in our numerical experiments corresponds to a triangular lattice of biskyrmion bubbles containing Bloch lines.

The paper is organized as follows. Analytical expressions for individual biskyrmion and biskyrmion lattices within the BP model are given in Sec. II. Biskyrmion lattices in a 2D magnetic film with PMA and DDI are studied numerically within a discrete spin model in Sec. III. Our results are summarized in Sec. IV.

\section{BISKYRMION LATTICES IN A 2D EXCHANGE MODEL}

The $2 \mathrm{D}$ exchange model is described by the energy

$$
\mathcal{H}_{e x}=\frac{J}{2} \int d x d y\left(\frac{\partial \mathbf{s}}{\partial x} \frac{\partial \mathbf{s}}{\partial x}+\frac{\partial \mathbf{s}}{\partial y} \frac{\partial \mathbf{s}}{\partial y}\right),
$$

where $\mathbf{s}$ is a three-component fixed-length spin field, $\mathbf{s}^{2}=1$, $J$ is the exchange constant, and summation over spin components $\alpha=x, y, z$ is performed. We choose uniform magnetization, $\mathbf{s}=(0,0,-1)$, in the negative $z$ direction at infinity. Spin-field configurations belong to homotopy classes characterized by the topological charge

$$
Q=\int \frac{d x d y}{4 \pi} \mathbf{s} \cdot \frac{\partial \mathbf{s}}{\partial x} \times \frac{\partial \mathbf{s}}{\partial y}
$$

that takes quantized values $Q=0, \pm 1, \pm 2, \ldots$. The value of $Q$ shows how many times the spin vector circumscribes the full body angle $4 \pi$ as the position vector covers the whole $x y$ plane.

In each homotopy class, the energy is minimized by $\mathbf{s}(x, y)$ satisfying

$$
\mathbf{s} \times \nabla^{2} \mathbf{s}=0,
$$

which means that the spins are collinear with the exchange field. In 2D, this is equivalent to

$$
\frac{\partial \mathbf{s}}{\partial x}= \pm \mathbf{s} \times \frac{\partial \mathbf{s}}{\partial y}, \quad \frac{\partial \mathbf{s}}{\partial y}=\mp \mathbf{s} \times \frac{\partial \mathbf{s}}{\partial x} .
$$

One recovers Eq. (3) by differentiating the first of these equations over $y$ and the second one over $x$, and subtracting one from the other. With the help of Eqs. (1) and (4), it is easy to obtain the relation between the exchange energy and the topological charge [14]:

$$
E=4 \pi J|Q| .
$$

Extremal spin configurations can be obtained by mapping the problem onto the complex plane, $z=x+i y$. In terms of the complex function

$$
\omega(z)=\frac{s_{x}+i s_{y}}{1-s_{z}},
$$

Eqs. (4) reduce to linear equations,

$$
\frac{\partial \omega}{\partial x}= \pm i \frac{\partial \omega}{\partial y},
$$

that are familiar Cauchy-Riemann (CR) conditions of the analyticity of the function. The corresponding spin configuration follow from the relations

$$
s_{x}+i s_{y}=\frac{2 \omega}{|\omega|^{2}+1}, \quad s_{z}=\frac{|\omega|^{2}-1}{|\omega|^{2}+1} .
$$

The remarkable property of the model is that any analytic function $\omega(z)$ provides a solution for the extremal spin configuration.

In particular, the sum of poles

$$
\omega(z)=\sum_{i} \frac{\lambda_{i} e^{i \gamma_{i}}}{z-z_{i}}
$$

describes a collection of antiskyrmions with spins at the poles pointing up against the spin-down background and the parameters $z_{i}, \lambda_{i}, \gamma_{i}$ defining the position, size, and chirality angle of the $i$ th antiskyrmion. This function satisfies the CR condition, Eq. (7), with the plus sign. In-plane spin components are rotating counterclockwise as the observation point is moving clockwise around the center (the pole) of the antiskyrmion. Each antiskyrmion is contributing -1 to the total topological charge $Q$.

The state with the skyrmions whose central spins are pointing down against the spin-up background can be obtained by rotating all spins of the above collection of antiskyrmions by the angle $\pi$ around the $x$ axis. Conformal invariance of the $2 \mathrm{D}$ exchange model allows one to achieve the same effect by taking the reciprocal of the $\omega$ function,

$$
\omega(z)=\left(\sum_{i} \frac{\lambda_{i} e^{i \gamma_{i}}}{z-z_{i}}\right)^{-1} .
$$

It describes a collection of skyrmions pointing down against the spin-up background, with each skyrmion corresponding to a zero of $\omega(z)$. Since the rotation of all spins preserves the homotopy class, such skyrmions have topological charge 


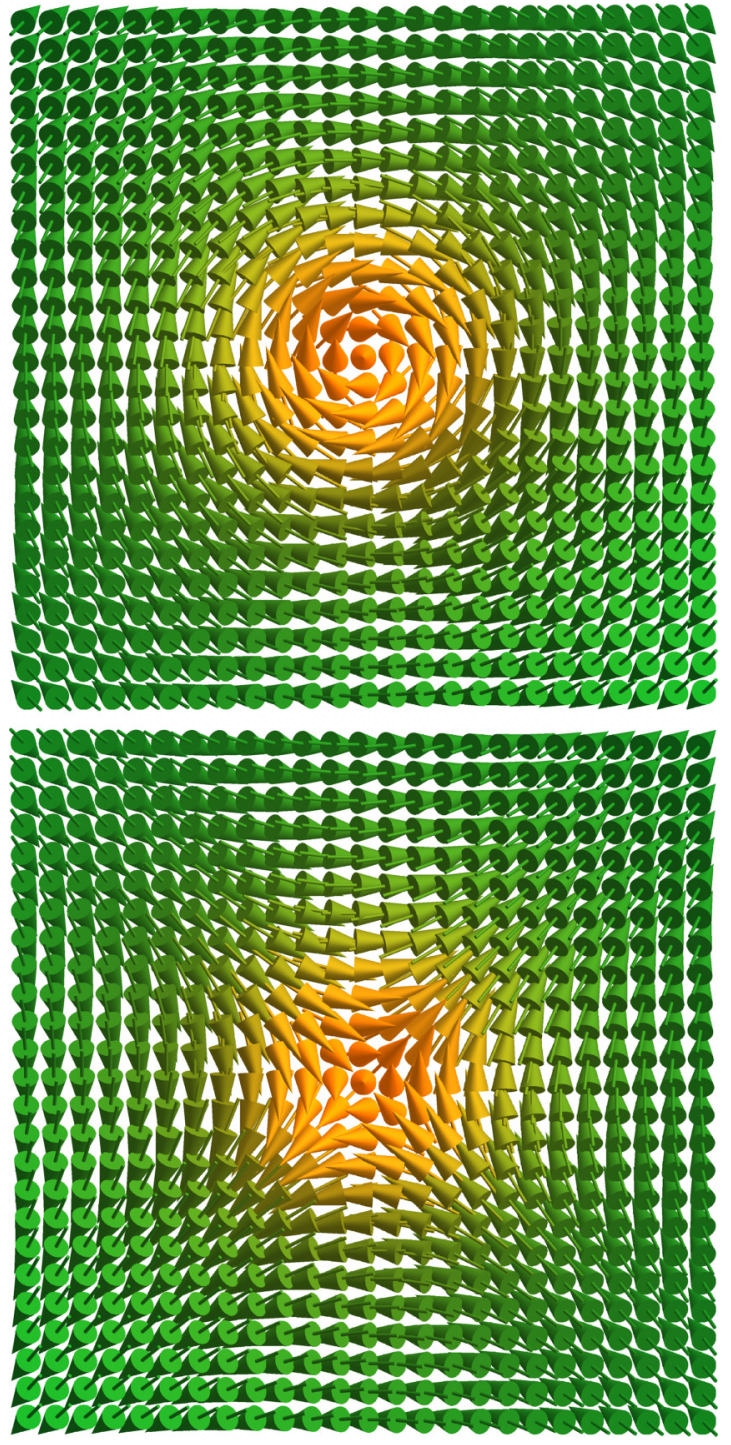

FIG. 2. Bloch-type skyrmion (upper pannel) and antiskyrmion (lower panel) spin-field configurations. In-plane components of the field rotate clockwise for the skyrmion and counterclockwise for the antiskyrmion as one goes clockwise around the center of the topological defect.

$Q=-1$, with in-plane spin components rotating clockwise when the observation point moves clockwise around the skyrmion's center. This illustrates a less appreciated fact that the sign of $Q$ is determined by both the topology of the solution and the boundary condition (direction of spins at infinity). The physical distinction between magnetic skyrmion and antiskyrmion is determined by the difference in their spin-field configurations illustrated in Fig. 2.

The expression with a complex-conjugated argument

$$
\omega(z)=\sum_{i} \frac{\lambda_{i} e^{i \gamma_{i}}}{z^{*}-z_{i}^{*}},
$$

that satisfies the CR condition Eq. (7) with the minus sign, describes a collection of skyrmions whose central spins are pointing up against the spin-down background. Here, each skyrmion has topological charge $Q=1$.
The energy of the collection of skyrmions or collection of antiskyrmions is entirely defined by the total topological charge $Q$ through Eq. (5). $Q$ is determined by the number of poles and is independent of $z_{i}, \lambda_{i}$, and $\gamma_{i}$ due to the symmetry of the $2 \mathrm{D}$ exchange model. In contrast, any combination of skyrmions and antiskyrmions yields a nonanalytical $\omega(z)$ that violates the CR conditions and hence does not satisfy Eq. (3). In the physical language, this means that such a configuration cannot be static. Skyrmions and antiskyrmions must annihilate while conserving the total topological charge $Q$, with the excess energy going into spin waves.

For an arbitrary $Q$, a skyrmion solution centered at $z=z_{0}$ is given by

$$
\omega(z)=\left(\frac{\lambda}{z^{*}-z_{0}^{*}}\right)^{Q} e^{i \gamma}
$$

The antiskyrmion solution is obtained by replacing $z^{*}$ and $z_{0}^{*}$ with $z$ and $z_{0}$. A biskyrmion with $Q=2$ and separation $d$ between two skyrmions of chirality $\gamma_{1}$ and $\gamma_{2}$ in the biskyrmion, centered at $z=0$, can be written as

$$
\omega(z)=\frac{\lambda e^{i \gamma_{1}}}{z^{*}+d / 2}+\frac{\lambda e^{i \gamma_{2}}}{z^{*}-d / 2} .
$$

A generic Bloch-type biskyrmion with opposite chiralities $\gamma_{1}=-\gamma_{2}=\pi / 2$ is shown in Fig. 1 .

Regular lattices of topological defects (skyrmions or antiskyrmions) can be constructed using functions that are double periodic in the complex plane $z=x+i y$. The elliptic Weierstrass $\wp$ function provides such a solution for the periodic lattice of $Q=2$ skyrmions,

$$
\begin{aligned}
\wp\left(z^{*}\right)= & \frac{1}{z^{* 2}}+\sum_{m, n \neq 0}\left[\frac{1}{\left(z^{*}+2 m p_{1}+2 n p_{2}\right)^{2}}\right. \\
& \left.-\frac{1}{\left(2 m p_{1}+2 n p_{2}\right)^{2}}\right] .
\end{aligned}
$$

Here $m, n$ are integers, and $p_{1,2}$ are half-periods of the elliptic function satisfying $\operatorname{Im}\left(p_{2} / p_{1}\right) \neq 0$. Consequently $\wp\left(z^{*}\right)=$ $\wp\left(z^{*}+2 m p_{1}\right)=\wp\left(z^{*}+2 n p_{2}\right)$, so that $Q=2$ skyrmions are found at $z^{*}=2 m p_{1}+2 n p_{2}$.

Following experimental observations, we will be interested in the triangular biskyrmion lattices. They correspond to the choice

$$
p_{1}=\frac{a^{\prime}}{2}, \quad p_{2}=\frac{-1+i \sqrt{3}}{4} a^{\prime},
$$

where $a^{\prime}$ is the skyrmion lattice spacing. The choice for the $\omega$ function is $\omega=\lambda^{2} e^{-i \gamma} \wp\left(z^{*}\right)$, where $\lambda$ and $\gamma$ represent the size and chirality of skyrmions in the lattice. Two such lattices with $\gamma=0$ and $\gamma=\pi / 2$ are shown in Fig. 3 .

So far the lattices we have built had zero separation $d$ between the two skyrmions in a $Q=2$ topological defect; see Eq. (13). To build biskyrmion lattices with a finite $d$, one can use the Weierstrass $\zeta$ function defined by 


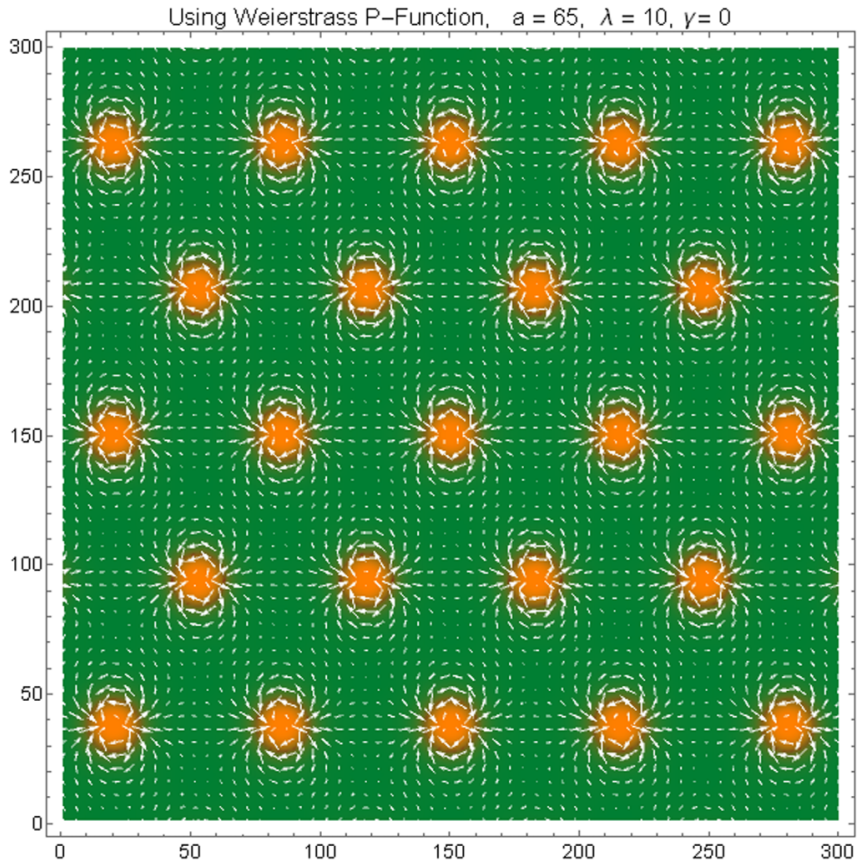

FIG. 3. Triangular $Q=2$ skyrmion lattices generated with the help of the Weierstrass $\wp$ function.

$d \zeta\left(z^{*}\right) / d z^{*}=-\wp\left(z^{*}\right)$. Its explicit form is given by

$$
\begin{aligned}
\zeta\left(z^{*}\right)= & \frac{1}{z^{*}}+\sum_{m, n \neq 0}\left[\frac{1}{z^{*}-2 n p_{1}-2 m p_{2}}\right. \\
& \left.+\frac{1}{2 n p_{1}+2 m p_{2}}+\frac{z^{*}}{\left(2 n p_{1}+2 m p_{2}\right)^{2}}\right] .
\end{aligned}
$$

This elliptic function is only quasiperiodic, satisfying $\zeta\left(z^{*}+\right.$ $\left.2 m p_{1}+2 n p_{2}\right)=\zeta\left(z^{*}\right)+2 m \zeta\left(p_{1}\right)+2 n \zeta\left(p_{2}\right)$. However, the function

$$
\omega\left(z^{*}\right)=\frac{\lambda^{2}}{d} e^{i \gamma}\left[\zeta\left(z^{*}+\frac{d}{2} e^{i \eta}\right)-\zeta\left(z^{*}-\frac{d}{2} e^{i \eta}\right)\right]
$$

is double periodic. It describes the lattice of biskyrmions of opposite chiralities that have a finite separation $d$ between skyrmions in a biskyrmion. The value of $\eta$ selects the orientation of the biskyrmion, with $\eta=0$ and $\eta=\pi / 2$ corresponding to the horizontal and vertical orientation respectively. Lattices with vertical orientation of Néel and Bloch biskyrmions are shown in Fig. 4.

It is interesting to notice that standard elliptic functions are well suited for the description of $Q=2$ skyrmion and biskyrmion lattices but not of $Q=1$ skyrmion lattices because elliptic functions with first-order poles on a periodic lattice do not exist [34]. By considering derivatives of $\wp$ functions, one can, in principle, construct lattices of skyrmions with $Q>2$. We are not considering them here because they have not been observed in experiment and because there is no reason to expect that such lattices would have energies lower than the energy of a biskyrmion lattice.

\section{BISKYRMION LATTICES IN A 2D MAGNETIC FILM}

In the numerical work, we study a lattice model of a ferromagnetic film of finite thickness with the energy given
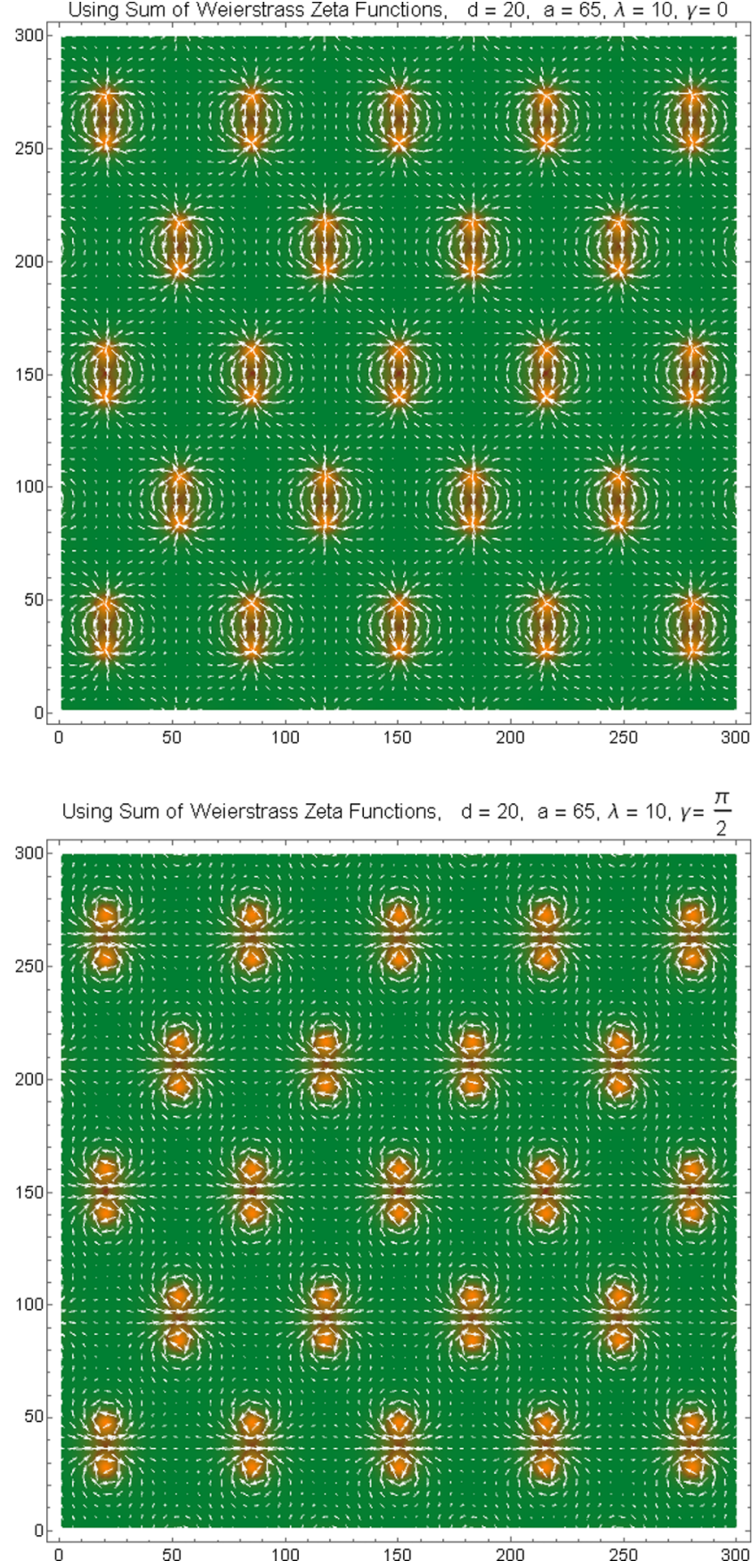

FIG. 4. Triangular biskyrmion lattices generated with the help of Weierstrass $\zeta$ functions. Upper panel: Néel biskyrmions, $\gamma=0$. Lower panel: Bloch biskyrmions, $\gamma=\pi / 2$.

by the sum over lattice sites $i, j$

$$
\begin{aligned}
\mathcal{H}= & -\frac{1}{2} \sum_{i j} J_{i j} \mathbf{s}_{i} \cdot \mathbf{s}_{j}-H \sum_{i} s_{i z}-\frac{D}{2} \sum_{i} s_{i z}^{2} \\
& -\frac{E_{D}}{2} \sum_{i j} \Phi_{i j, \alpha \beta} s_{i \alpha} s_{j \beta} .
\end{aligned}
$$

Here, the exchange coupling is $J$ for the nearest neighbors on a simple cubic lattice and zero otherwise, $D$ is the easy-axis 
PMA constant, and $H \equiv g \mu_{B} S B$, with $S$ being the value of the atomic spin and $B$ being the induction of the applied magnetic field. In the DDI part of the energy,

$$
\Phi_{i j, \alpha \beta} \equiv a^{3} r_{i j}^{-5}\left(3 r_{i j, \alpha} r_{i j, \beta}-\delta_{\alpha \beta} r_{i j}^{2}\right),
$$

where $\mathbf{r}_{i j} \equiv \mathbf{r}_{i}-\mathbf{r}_{j}$ is the displacement vector between the lattice sites and $\alpha, \beta=x, y, z$ denote Cartesian components in a three-dimensional (3D) coordinate space. The parameter $E_{D}=\mu_{0} M_{0}^{2} a^{3} /(4 \pi)$ defines the strength of the DDI, with $M_{0}=g \mu_{B} S / a^{3}$ being the magnetization for our lattice model and $\mu_{0}$ being the magnetic permeability of vacuum.

The ratio of the PMI and DDI is given by the dimensionless parameter $\beta \equiv D /\left(4 \pi E_{D}\right)$. For $\beta>1$, the energy of the uniform state with spins directed along the $z$ axis is lower than that of the state with spins lying in the film's plane. For $\beta<1$, the state with spins in the plane has a lower energy. The most interesting practical case is $\beta \sim 1$ realized in many materials due to considerable compensation of the effects of the PMA and DDI.

An important parameter controlling the DDI is the film thickness represented by $N_{z}$ in the units of the atomic spacing $a$. For thin films that are studied here, the magnetization inside the film is nearly constant along the direction perpendicular to the film. Thus, one can make the problem effectively two-dimensional by introducing the effective DDI between the columns of parallel spins, considered as effective spins of the 2D model. This greatly speeds up the computation. To this end, for the simple cubic lattice, one can write the dipolar coupling, Eq. (19), as $\Phi_{i j, \alpha \beta}=\phi_{\alpha \beta}\left(n_{x}, n_{y}, n_{z}\right)$, where $n_{x} \equiv i_{x}-j_{x}$, etc., are the distances on the lattice and

$$
\phi_{\alpha \beta}\left(n_{x}, n_{y}, n_{z}\right)=\frac{3 n_{\alpha} n_{\beta}-\delta_{\alpha \beta}\left(n_{x}^{2}+n_{y}^{2}+n_{z}^{2}\right)}{\left(n_{x}^{2}+n_{y}^{2}+n_{z}^{2}\right)^{5 / 2}} .
$$

The effective DDI is defined by

$$
\bar{\phi}_{\alpha \beta}\left(n_{x}, n_{y}\right)=\frac{1}{N_{z}} \sum_{i_{z}, j_{z}=1}^{N_{z}} \phi_{\alpha \beta}\left(n_{x}, n_{y}, i_{z}-j_{z}\right) .
$$

Using the symmetry, one can express this result in the form with only one summation,

$$
\begin{aligned}
\bar{\phi}_{\alpha \beta}\left(n_{x}, n_{y}\right)= & \phi_{\alpha \beta}\left(n_{x}, n_{y}, 0\right) \\
& +\frac{2}{N_{z}} \sum_{n_{z}=1}^{N_{z}-1}\left(N_{z}-n_{z}\right) \phi_{\alpha \beta}\left(n_{x}, n_{y}, n_{z}\right),
\end{aligned}
$$

that is used in the computations. That effective DDI (that can be precomputed) has different forms in different ranges of the distance $r$. At $r \gtrsim a N_{z}$, it scales as the interaction of magnetic dipoles $1 / r^{3}$, while at $r \lesssim a N_{z}$ it goes as $1 / r$ that corresponds to the interaction of magnetic charges at the surface of the film.

Starting with a biskyrmion lattice of the previous section as an initial condition, we compute its evolution toward the minimum-energy configuration in a system containing up to $1000 \times 1000 \times 100$ spins. Our numerical method [35] combines sequential rotations of spins $\mathbf{s}_{i}$ towards the direction of the local effective field, $\mathbf{H}_{\mathrm{eff}, i}=-\partial \mathcal{H} / \partial \mathbf{s}_{i}$, with the probability $\alpha$, and the energy-conserving spin flips (so-called over-relaxation $), \mathbf{s}_{i} \rightarrow 2\left(\mathbf{s}_{i} \cdot \mathbf{H}_{\mathrm{eff}, i}\right) \mathbf{H}_{\mathrm{eff}, i} / H_{\mathrm{eff}, i}^{2}-\mathbf{s}_{i}$, with the probability $1-\alpha$. The parameter $\alpha$ plays the role of the effective relaxation constant. We mainly use the value $\alpha=0.03$ that provides the overall fastest convergence.

The dipolar part of the effective field takes the longest time to compute. The most efficient method uses updates of the dipolar field after all spins are updated rather than after updating each individual spin. Computation of the dipolar field uses the fast Fourier transform (FFT) algorithm that yields the dipolar fields in the whole sample as one program step. The total topological charge $Q$ of the lattice is computed numerically using the lattice-discretized version of Eq. (2) in which first derivatives are approximated by the four-point formula.

An alternative way to minimize the energy can be a numerical solution of the damped Landau-Lifshitz equation. While doing this, one can either keep or discard the precession terms, with the latter method being a variant of the gradient flow method as the spins in each step would be rotating strictly in the directions that maximize the decrease of energy. This method is parallel rather than sequential because all spins are updated in one integration step. The computational method used by us (field alignment with over-relaxation) proves to be faster.

Computations were performed with Wolfram Mathematica using compilation. Most of the numerical work has been done on the 20-core Dell Precision T7610 Workstation. The FFT for computing the DDI was performed via Mathematica's function ListConvolve that implicitly uses many processor cores. For this reason, no explicit parallelization was done in our program.

For each skyrmion state, we compute the topological charge $Q$, the exchange energy $E_{e x}$, the total energy $E$, and the difference, $\Delta E$, of the total energy from the energy of the uniformly magnetized state. The difference of the exchange energy from $4 \pi J Q$ is the measure of the distortion of $\mathrm{BP}$ biskyrmions, while the sign of $\Delta E$ is indicative of whether the formation of the biskyrmion lattice can lower the total energy. Our numerical results for the energies are presented in the units of $J$.

Typical results of the energy minimization at $H=0$ and $\beta=1$ for two values of PMA, starting with the same Weierstrass biskyrmion lattice as the initial condition, are illustrated by Figs. 5 and 6 . The final state does not depend on the separation $d$ in the biskyrmions but depends on the lattice period which defines the total topological charge of the system. The latter is approximately conserved and changes only slightly due to the use of the open boundaries. At low PMA, $D / J=0.001$, the system relaxes to a biskyrmion lattice shown in Fig. 5. Its energy, however, is above the energy of the uniformly magnetized state, $\Delta E>0$, indicating that it can only be a metastable state stabilized by the conservation of the topological charge.

At $D / J=0.03$, the initial Weierstrass biskyrmion lattice evolves into a lattice of biskyrmion bubbles shown Fig. 6. The energy of such a lattice is below the energy of the uniformly magnetized state, $\Delta E<0$, indicating that it can be a stable domain structure at $H=0$. It consists of regularly spaced magnetic bubbles with $Q=2$, each having two Bloch lines. Notice that Bloch lines in biskyrmion bubbles forming a triangular lattice at $H=0$ have been observed in experiment [24]. 


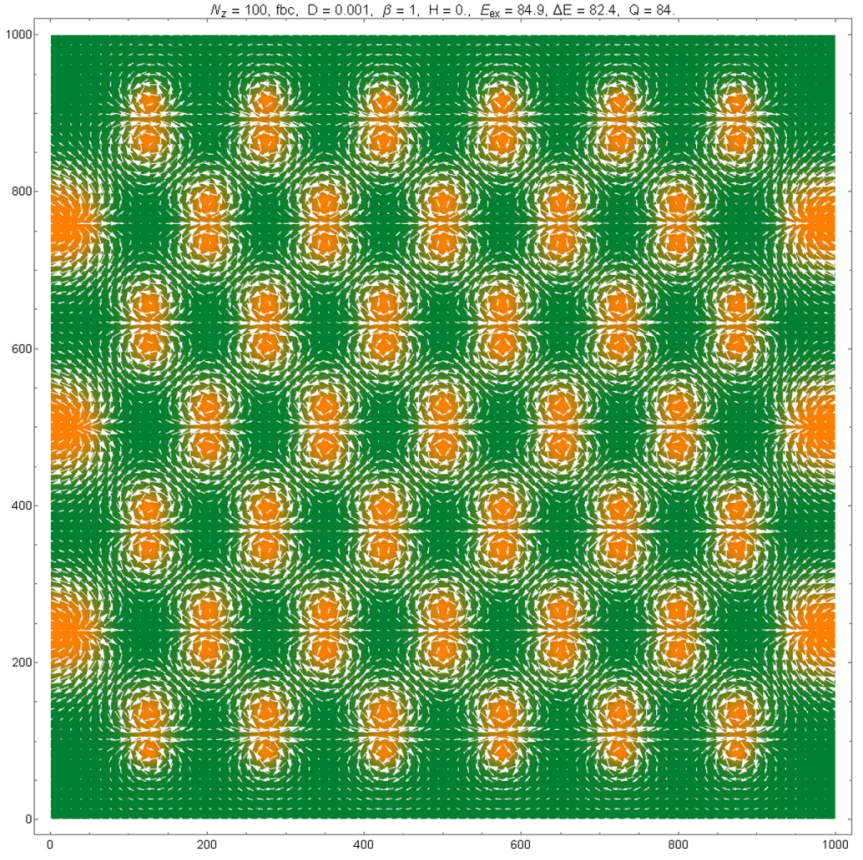

FIG. 5. Biskyrmion lattice that evolves from the Weierstrass lattice similar to the one shown in the lower panel of Fig. 4 under the action of PMA and DDI at $H=0, \beta=1$, and $D=0.001$. The energy of this state is higher than the energy of the uniformly magnetized state, $\Delta E=82.4$.

Even a lower energy can be achieved by increasing the period of the lattice, which leads to bigger biskyrmion bubbles in the final state; see Fig. 7. This finding confirms conjecture

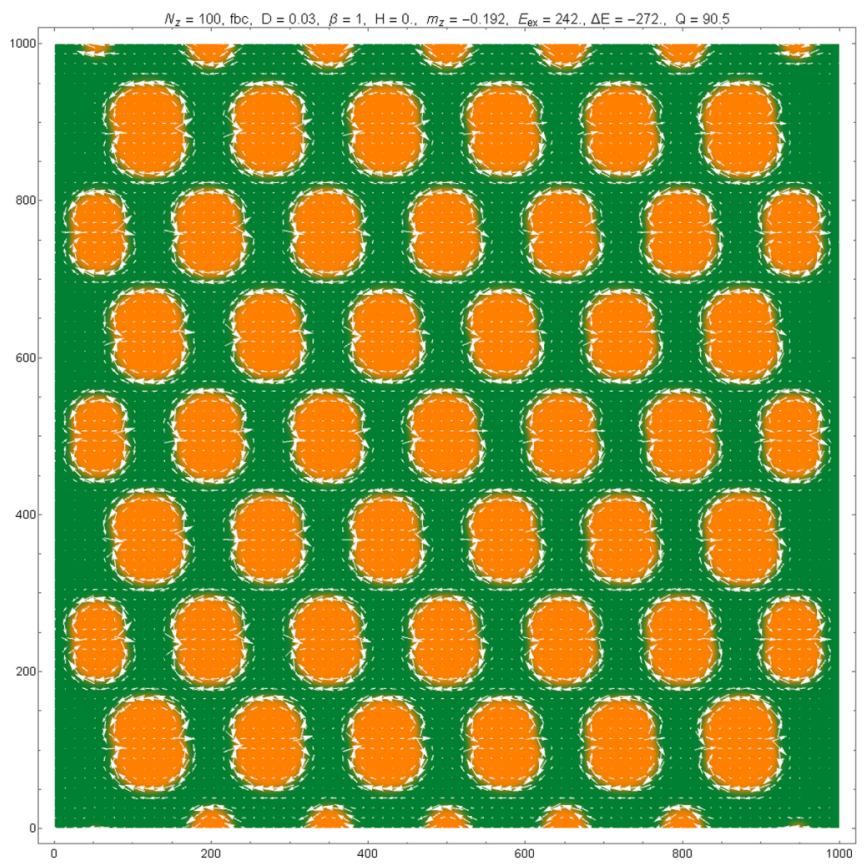

FIG. 6. Biskyrmion bubble lattice that evolves from the Weierstrass lattice under the action of PMA and DDI at $H=0, \beta=1$, and $D=0.03$. Its energy is lower than the energy of the uniformly magnetized state, $\Delta E=-272$, indicating that it can be a stable domain structure formed by the DDI.

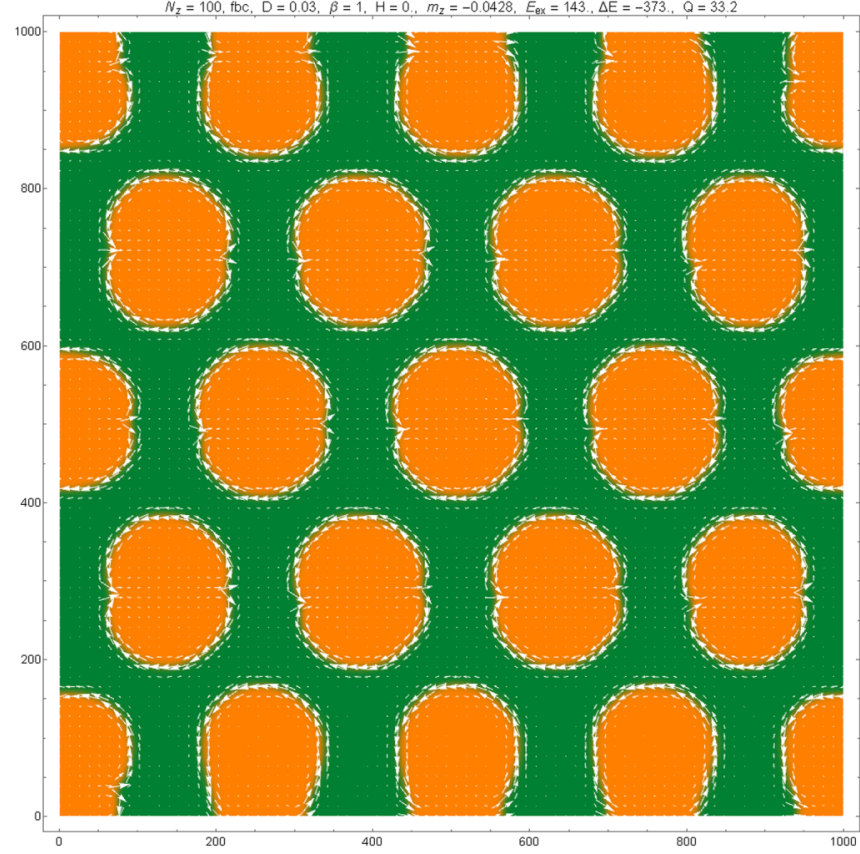

FIG. 7. Biskyrmion bubble lattice with lower energy, $\Delta E=$ -373 , that evolves from the Weierstrass lattice of longer period under the action of PMA and DDI at $H=0, \beta=1$, and $D=0.03$.

of Ref. [26] that the absolute minimum of the energy is likely to correspond to the limiting case of biskyrmion bubbles of size much greater than the domain wall thickness.

\section{DISCUSSION}

We have studied the complex-plane representation of skyrmion and antiskyrmion spin configurations. Our focus has been on biskyrmion lattices in nonchiral magnetic films of finite thickness. While such lattices have been observed in experiments, their theoretical description presented a considerable challenge. Our approach has been based upon observation that representation of ferromagnetically coupled spins that uses a complex function $\omega(z)=\left(s_{x}+i s_{y}\right) /\left(1-s_{z}\right)$ of $z=x+i y$, naturally provides biskyrmion lattices when $\omega(z)$ is written in terms of elliptic functions. Starting with such a function as an initial condition, we study evolution of the spin configuration under the action of perpendicular magnetic anisotropy (PMA) and dipole-dipole interaction (DDI).

Our main result is that in a certain a range of parameters, that is relevant to experimental situations, the energy of a triangular lattice of biskyrmion bubbles is significantly lower than the energy of the uniformly magnetized film in a zero magnetic field. Notice in this connection that for a large, highly nonlinear, hysteretic magnetic system that has infinite number of local energy minima one cannot find and compare energies of all metastable nonuniformly magnetized states that emerge due to the exchange interaction, PMA, and DDI (such as various kinds of labyrinth domains, various kinds of cylindrical domains or skyrmions, with and without Bloch lines and Bloch points, etc.). Consequently, it is impossible to say with the absolute certainty that a biskyrmion lattice observed in experiments and simulations is the ground state of 
the system. Its low energy computed in our model, however, makes it plausible that it represents a kind of the energyminimizing domain structure that has not been observed until recently.

When biskyrmion lattices correspond to the energy minimum, they are likely to consist of bubbles of size much greater than the domain wall thickness. An additional connection between theory and observation is provided by the fact that biskyrmions arranged in a triangular lattice possess Bloch lines in both experiments and computations. Further experimental studies of magnetic phases of nonchiral films along the hysteresis loop will shed more light on the place of biskyrmion lattices in the phase diagram.

\section{ACKNOWLEDGMENT}

This work has been supported by Grant No. DE-FG0293ER45487 funded by the U.S. Department of Energy, Office of Science.
[1] T. H. R. Skyrme, A non-linear theory of strong interactions, Proc. R. Soc. London, Ser. A 247, 260 (1958).

[2] A. M. Polyakov, Gauge Fields and Strings (Harwood Academic, Chur, Switzerland, 1987).

[3] N. Manton and P. Sutcliffe, Topological Solitons (Cambridge University Press, Cambridge, UK, 2004).

[4] E. Braaten and L. Carson, Deuteron as a toroidal skyrmion, Phys. Rev. D 38, 3525 (1988).

[5] W. Y. Crutchfield, N. J. Snyderman, and V. R. Brown, Deuteron in the Skyrme Model, Phys. Rev. Lett. 68, 1660 (1992).

[6] N. Nagaosa and Y. Tokura, Topological properties and dynamics of magnetic skyrmions, Nat. Nanotechnol. 8, 899 (2013).

[7] X. Zhang, M. Ezawa, and Y. Zhou, Magnetic skyrmion logic gates: Conversion, duplication, and merging of skyrmions, Sci. Rep. 5, 9400 (2015).

[8] G. Finocchio, F. Büttner, R. Tomasello, M. Carpentieri, and M. Klaui, Magnetic skyrmions: From fundamental to applications, J. Phys. D 49, 423001 (2016).

[9] A. O. Leonov, T. L. Monchesky, N. Romming, A. Kubetzka, A. N. Bogdanov, and R. Wiesendanger, The properties of isolated chiral skyrmions in thin magnetic films, New J. Phys. 18, 065003 (2016).

[10] W. Jiang, G. Chen, K. Liu, J. Zang, S. G. E. te Velthuis, and A. Hoffmann, Skyrmions in magnetic multilayers, Phys. Rep. 704, 1 (2017).

[11] A. Fert, N. Reyren, and V. Cros, Magnetic skyrmions: Advances in physics and potential applications, Nat. Rev. Mater. 2, 17031 (2017).

[12] A. P. Malozemoff and J. C. Slonczewski, Magnetic Domain Walls in Bubble Materials (Academic Press, New York, 1979).

[13] T. H. O'Dell, Ferromagnetodynamics: The Dynamics of Magnetic Bubbles, Domains, and Domain Walls (Wiley, New York, 1981).

[14] A. A. Belavin and A. M. Polyakov, Metastable states of twodimensional isotropic ferromagnets, Pis'ma Zh. Eksp. Teor. Fiz. 22, 503 (1975) [JETP Lett. 22, 245 (1975)].

[15] E. M. Chudnovsky and J. Tejada, Lectures on Magnetism (Rinton Press, Princeton, NJ, 2006).

[16] M. Ezawa, Giant Skyrmions Stabilized by Dipole-Dipole Interactions in Thin Ferromagnetic Films, Phys. Rev. Lett. 105, 197202 (2010).

[17] I. Makhfudz, B. Kröger, and O. Tchernyshyov, Inertia and Chiral Edge Modes of a Skyrmion Magnetic Bubble, Phys. Rev. Lett. 109, 217201 (2012).
[18] L. Cai, E. M. Chudnovsky, and D. A. Garanin, Collapse of skyrmions in two-dimensional ferromagnets and antiferromagnets, Phys. Rev. B 86, 024429 (2012).

[19] U. K. Rößler, N. Bogdanov, and C. Pfleiderer, Spontaneous skyrmion ground states in magnetic metals, Nature (London) 442, 797 (2006).

[20] S. Heinze, K. von Bergmann, M. Menzel, J. Brede, A Kubetzka, R. Wiesendanger, G. Bihlmayer, and S. Blugel, Spontaneous atomic-scale magnetic skyrmion lattice in two dimensions, Nat. Phys. 7, 713 (2011).

[21] O. Boulle, J. Vogel, H. Yang, S. Pizzini, D. de Souza Chaves, A. Locatelli, T. O. Mentes, A. Sala, L. D. Buda-Prejbeanu, O. Klein et al., Room-temperature chiral magnetic skyrmions in ultrathin magnetic nanostructures, Nat. Nanotechnol. 11, 449 (2016).

[22] E. M. Chudnovsky and D. A. Garanin, Topological Order Generated by a Random Field in a 2D Exchange Model, Phys. Rev. Lett. 121, 017201 (2018).

[23] E. M. Chudnovsky and D. A. Garanin, Skyrmion glass in a 2D Heisenberg ferromagnet with quenched disorder, New J. Phys. 20, 033006 (2018).

[24] X. Z. Yu, Y. Tokunaga, Y. Kaneko, W. Z. Zhang, K. Kimoto, Y. Matsui, Y. Taguchi, and Y. Tokura, Biskyrmion states and their current-driven motion in a layered manganite, Nat. Commun. 5, 3198 (2014).

[25] W. Wang, Y. Zhang, G. Xu, L. Peng, B. Ding, Y. Wang, Z. Hou, X. Zhang, X. Li, E. Liu et al., A centrosymmetric hexagonal magnet with superstable biskyrmion magnetic nanodomains in a wide temperature range of 100-340 K, Adv. Mater. 28, 6887 (2016).

[26] J. C. Loudon, A. C. Twitchett-Harrison, D. Cortés-Ortuño, M. T. Birch, L. A. Turnbull, A. Štefančič, F. Y. Ogrin, E. O. BurgosParra, N. Bukin, A. Laurenson et al., Do images of biskyrmions show type-II bubbles?, Adv. Mater. 31, 1806598 (2019).

[27] Y. Yao, B. Ding, J. Cui, X. Shen, Y. Wang, W. Wang, and R. Yu, Magnetic hard nanobubble: A possible magnetic structure behind the bi-skyrmion, Appl. Phys. Lett. 114, 102404 (2019).

[28] B. Göbel, J. Henk, and I. Mertig, Forming individual magnetic biskyrmions by merging two skyrmions in a centrosymmetric nanodisk, Sci. Rep. 9, 9521 (2019).

[29] D. A. Garanin, E. M. Chudnovsky, and X. X. Zhang, Skyrmion clusters from Bloch lines in ferromagnetic films, Europhys. Lett. 120, 17005 (2017).

[30] X. Zhang, J. Xia, Y. Zhou, X. Liu, and M. Ezawa, Skyrmions and antiskyrmions in a frustrated $J_{1}-J_{2}-J_{3}$ ferromagnetic film: 
Current-induced helicity locking-unlocking transition, Nat. Commun. 8, 1717 (2017).

[31] A. O. Leonov and M. Mostovoy, Multiply periodic states and isolated skyrmions in an anisotropic frustrated magnet, Nat. Commun. 6, 8275 (2015).

[32] S.-Z. Lin and S. Hayami, Ginzburg-Landau theory for skyrmions in inversion-symmetric magnets with competing interactions, Phys. Rev. B 93, 064430 (2016).
[33] L. Rózsa, K. Palotás, A. Deák, E. Simon, R. Yanes, L. Udvardi, L. Szunyogh, and U. Nowak, Formation and stability of metastable skyrmionic spin structures with various topologies in an ultrathin film, Phys. Rev. B 95, 094423 (2017).

[34] N. I. Akhiezer, Elements of the Theory of Elliptic Functions, Translations of Mathematical Monographs Vol. 79 (American Mathematical Society, Providence, RI, 1990).

[35] D. A. Garanin, E. M. Chudnovsky, and T. Proctor, Random field xy model in three dimensions, Phys. Rev. B 88, 224418 (2013). 